cuing memory during sleep is an appealing approach. Bendor and Wilson's results ${ }^{6}$, in the context of extensive research on memory modification during sleep ${ }^{1,3,10,11}$, describe ways and mechanisms by which memories can be manipulated while they consolidate during sleep to enhance some and to indirectly weaken others. Techniques of selective enhancement of desired memories and indirect suppression of unwanted memories could find applications not only in education (for example, to strengthen vocabulary in language students), but also in therapeutic settings (for example, to fade traumatic memories in PTSD patients).

Beyond applications, Bendor and Wilson's approach to cuing memory reactivations during sleep provokes a question of primary scientific interest. If cuing induces the brain to reactivate a specific memory during sleep, then what are the factors determining which memory is reactivated spontaneously under natural conditions? Considering memory consolidation during sleep as an active and selective process occurring under conditions of limited capacity leads to the question of what is telling the hippocampus which memory to reactivate and enhance during sleep and which to not. This question cannot be answered by the cuing-during-sleep approach. Nevertheless, Bendor and Wilson's finding provide some clues as to the kind of communication that comes into play. The auditory cues that were effective in biasing memory replay were presumably processed in the neocortex before this information reached the hippocampus, and they did not induce hippocampal replay when presented during waking - presumably owing to the different context of presentation (that is, away from the task, in a separate cage). These findings converge with evidence that, during natural sleep, the neocortical EEG slow oscillations that are a hallmark of slow-wave sleep synchronize hippocampal replay to periods of increased brain excitability ${ }^{10,12,13}$. In telling the hippocampus when to reactivate, slow oscillations coming from the neocortex might also provide information as to what to reactivate. But so far this is mere speculation. In the context of Bendor and Wilson's ${ }^{6}$ approach of forced memory reactivation, understanding how neocortex and hippocampus interact in spontaneous conditions to select memories for consolidation appears to be a corollary and most challenging question in this field.

\section{COMPETING FINANCIAL INTERESTS}

The authors declare no competing financial interests.

1. Rasch, B., Büchel, C., Gais, S. \& Born, J. Science 315 1426-1429 (2007).

2. Diekelmann, S., Büchel, C., Born, J. \& Rasch, B. Nat. Neurosci. 14, 381-386 (2011).

3. Antony, J.W., Gobel, E.W., O'Hare, J.K., Reber, P.J. \& Paller, K.A. Nat. Neurosci. 15, 1114-1116 (2012).

4. Diekelmann, S. \& Born, J. Nat. Rev. Neurosci. 11 , 114-126 (2010).

5. Wilson, M.A. \& McNaughton, B.L. Science 265 676-679 (1994).

6. Bendor, D. \& Wilson, M.A. Nat. Neurosci. 15, 1439-1444 (2012).

7. Girardeau, G., Benchenane, K., Wiener, S.I., Buzsáki, G. \& Zugaro, M.B. Nat. Neurosci. 12, 1222-1223 (2009).

8. Ego-Stengel, V. \& Wilson, M.A. Hippocampus 20, 1-10 (2010).

9. Tononi, G. \& Cirelli, C. Sleep Med. Rev. 10, 49-62 (2006).

10. Marshall, L., Helgadottir, H., Mölle, M. \& Born, J. Nature 444, 610-613 (2006).

11. Rudoy, J.D., Voss, J.L., Westerberg, C.E. \& Paller, K.A. Science 326, 1079 (2009).

12. Sirota, A., Csicsvari, J., Buhl, D. \& Buzsáki, G. Proc. Natl. Acad. Sci. USA 100, 2065-2069 (2003).

13. Ji, D. \& Wilson, M.A. Nat. Neurosci. 10, 100-107 (2007).

\title{
Changing behavior with epigenetics
}

Honeybees in a hive have different fixed roles, or castes, even though their DNA sequence is often identical. What then decides these differing roles and are there any epigenetic changes that parallel these differences? On page 1371, Herb and colleagues provide some answers to these questions.

It is already known that a deciding factor for the role assigned to a honeybee is its care early in life: female larvae that are fed more royal jelly (a protein-rich secretion from glands in worker bees' heads) grow into queen bees, whereas larvae destined to be worker bees only get limited amounts of this secretion. Queen bees are in charge of laying eggs, whereas worker bees take on differing roles at different ages, ranging from cleaning cells to carrying water.

Such differences in phenotype on the basis of early-life care are suggestive of an epigenetic influence. Accordingly, the authors used whole-genome bisulfite sequencing and high-throughput methylation analysis to look at differences in DNA methylation between sister bees.

Herb and colleagues found no DNA methylation differences between newly emerging worker and queen bees, but did uncover differences between two worker subcastes: nurse bees, which feed other bees, and foragers, which travel outside of the hive to collect food. Although the queen bee's behavior is fixed, and it cannot switch to being a worker, the roles of these worker subcastes are not fixed, and nurse bees can switch to foraging later in life. The transition from nurse to forager bee showed a relationship to DNA methylation, and the authors found over 150 methylated regions that were different in nurses and foragers.

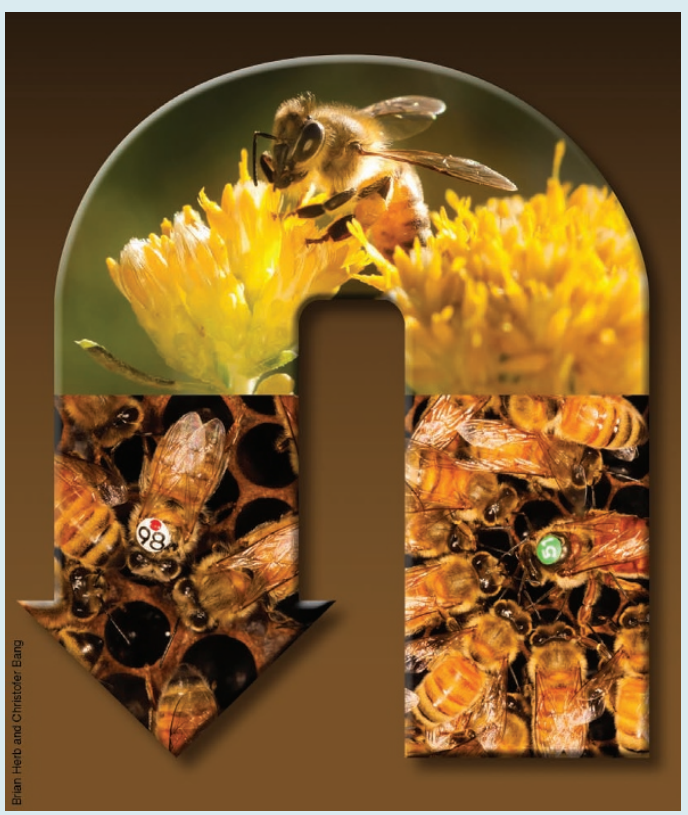

However, these differences could just be linked to the change from nurse to forager bee, rather than reflecting the differences in behavior between these subcastes. To exclude this possibility, the authors tricked some foragers into reverting back to their nurse roles. This behavioral 'U turn' resulted in DNA methylation patterns that were also mostly reverting, showing a pattern more like that seen in nurse bees than that in foragers.

These findings suggest that there is a close link between the pattern of DNA methylation and the role of worker bees. More broadly, these results underline that it is not just the DNA sequence that decides how genes modulate behavior and that epigenetic modulation can be a powerful effector of behavioral patterns.

Charvy Narain 